\title{
Molecular Identification and Polymorphism Determination of Cutaneous and Visceral Leishmaniasis Agents Isolated from Human and Animal Hosts in Iran
}

\author{
Homa Hajjaran, ${ }^{1}$ Mehdi Mohebali, ${ }^{1,2}$ Setareh Mamishi, ${ }^{3}$ \\ Farzaneh Vasigheh, ${ }^{1}$ Mohammad Ali Oshaghi, ${ }^{4}$ Saied Reza Naddaf, ${ }^{5}$ \\ Aref Teimouri, ${ }^{1}$ Gholam Hossein Edrissian, ${ }^{1}$ and Zabiholah Zarei ${ }^{6}$ \\ ${ }^{1}$ Department of Medical Parasitology and Mycology, School of Public Health, Tehran University of Medical Sciences, \\ Tehran 14155 6446, Iran \\ ${ }^{2}$ Center for Research of Endemic Parasites of Iran (CREPI), Tehran University of Medical Sciences, Tehran, Iran \\ ${ }^{3}$ Pediatric Infections Research Center, Tehran University of Medical Sciences, Tehran, Iran \\ ${ }^{4}$ Department of Medical Entomology and Vector Control, School of Public Health, Tehran University of Medical Sciences, Tehran, Iran \\ ${ }^{5}$ Department of Parasitology, Pasteur Institute of Iran, Tehran, Iran \\ ${ }^{6}$ School of Public Health, Meshkin-Shahr Research Station, Tehran University of Medical Sciences, Iran
}

Correspondence should be addressed to Homa Hajjaran; hajaranh@tums.ac.ir and Mehdi Mohebali; mohebali@tums.ac.ir

Received 25 April 2013; Revised 27 July 2013; Accepted 6 August 2013

Academic Editor: Georgios Theodoropoulos

Copyright (c) 2013 Homa Hajjaran et al. This is an open access article distributed under the Creative Commons Attribution License, which permits unrestricted use, distribution, and reproduction in any medium, provided the original work is properly cited.

\begin{abstract}
Amplification of internal transcript spacer 1 of ribosomal RNA (ITS1-RNA) gene followed by RFLP analysis and sequencing was used to identify the causing agents of cutaneous and visceral leishmaniasis (CL and VL) in humans and animal reservoir hosts from various geographical areas in Iran. We also used random amplified polymorphic DNA (RAPD-PCR) to obtain polymorphisms among isolates of Leishmania spp. Totally, 362 suspected human and animal cases including 173 CL, 49 VL, 60 rodents, and 80 domestic dogs were examined for Leishmania infection. From 112 culture-positive samples prepared from CL cases, 75 (67\%) were infected with L. major and 37 (33\%) with L. tropica. Of the 60 rodents examined, 25 (41.6\%) harbored the Leishmania infection; 21 were infected with $L$. major and 4 with L. turanica. From 49 suspected VL, 29 were positive by direct agglutination test (DAT), whereas microscopy detected parasite in bone marrow of 25 and culture in 28 of the patients. Two VL patients were infected with $L$. tropica and 26 with $L$. infantum. Of the 80 domestic dogs, 56 showed anti-Leishmania antibodies with DAT. Of these, 55 were positive by both microscopy and culture. Molecular identity, obtained only for 47 samples, revealed L. infantum in 43 and L. tropica in 4 dogs. The polymorphisms among L. tropica and L. major isolates were $3.6 \%$ and $7.3 \%$; the rate among human and canine VL isolates was $2.8 \%$ and $9.8 \%$, respectively. Our results showed that at least four different Leishmania species with various polymorphisms circulate among humans and animal hosts in Iran.
\end{abstract}

\section{Introduction}

Leishmaniases are a group of diseases caused by the parasites belonging to the genus Leishmania. The disease affects 98 tropical and Mediterranean countries, and 1.5 to 2 million cases are estimated to occur annually worldwide, with up to 350 million people at risk of infection [1]. Leishmaniases present a wide spectrum of clinical manifestations including cutaneous (CL), diffuse cutaneous (DCL), mucocutaneous
(MCL), and potentially fatal visceral leishmaniasis (VL), also known as "Kala-Azar," that are largely caused by different Leishmania species. In old world, three species caused CL: $L$. tropica, L. major (both usually cause localized skin lesions), and $L$. aethiopica that cause nonulcerative disseminated cutaneous lesions. VL is caused by parasites of the $L$. donovani-L. infantum complex. Recently, $L$. tropica has been implicated in several human cases with VL manifestations [2]. In Iran, the etiological agents of anthroponotic CL (ACL) 
and zoonotic CL (ZCL) are known to be L. tropica and $L$. major, respectively. L. infantum, the principal agent of $\mathrm{VL}$, causes splenomegaly and hepatomegaly [3]. Most of ACL are reported from northeastern and central parts of Iran [46 ], while ZCL extends from northeast to center and west of Iran and covers all southern provinces as well [7-9]. More than $90 \%$ of the visceral cases in Iran are reported from the northwest and some southern provinces including Fars and Bushehr [10]. All the three species of Leishmania could be found in some areas like Fars province in the south [11]. In Iran, the main reservoir hosts for ACL are human infected patients, for ZCL are some of the desert rodents particularly the great gerbils (Rhombomys opimus), and for VL are domestic and wild canines (mainly domestic dogs) $[4,12-15]$. In addition to the classic clinical leishmaniasis manifestations, there is a variety of atypical signs in CL including lupoid, mucosal lesions, and disseminated forms and in VL including symptomatic and asymptomatic forms [16-18]. The diagnosis of clinical forms of leishmaniasis is commonly based on visualization of the parasite in Giemsastained smears by microscopy and culture. Despite sensitivity and specificity, such methods fail to identify the infecting Leishmania species $[19,20]$. Over the last two decades, DNAbased approaches including PCR followed by sequencing or RFLP analysis and species-specific PCR have been widely used for the identification of Leishmania species [20-23].

Our study aimed to identify different Leishmania species in some foci of Iran and obtain polymorphisms among the species using RAPD-PCR.

\section{Materials and Methods}

\subsection{Sampling}

2.1.1. Cutaneous Leishmaniasis. Lesion aspirates were obtained from 173 suspected CL patients referred either to leishmaniasis laboratory at the School of Public Health, Tehran University of Medical Sciences (TUMS), or district health centers (DHCs) and hospitals in various regions of Iran from 2004 to 2012. Most patients were from northern, northeastern, western, central, and southern areas of the country. Samples were collected by injecting $0.2 \mathrm{~mL}$ of sterile saline into the dermis of the internal border of skin lesions with sterile insulin syringes. After suction, the sample was transferred to RPMI-1640 medium culture and some portion was checked for Leishmania infection by microscopy following the preparation of Giemsa-stained smears [22].

2.1.2. Rodents. Sixty rodents including 36 Rhombomys opimus, 5 Meriones libycus, 13 Nesokia indica, 5 Tatera indica, and one Mus musculus were trapped by live baits traps from $\mathrm{CL}$ endemic areas in the northeast and west of Iran. None of the animals had any acute cutaneous lesion. For preparation of samples, the external edges of the ear lobes were cut with scissors after washing and disinfection, and then low amounts of the exudates were transferred to culture media [24]. Giemsa-stained smears were prepared from the same samples.
2.1.3. Visceral Leishmaniasis. Blood samples were taken from 49 suspected VL cases, presented with fever, weakness, and hepatosplenomegaly, at the medical health centers or leishmaniasis laboratory at the School of Public Health (SPH), TUMS. Most patients were from northwestern, western, central, and southern areas of the country. Also, the bone marrow aspirate was taken from iliac of the same patients under local anesthesia by physicians.

Also, blood samples were taken from eighty dogs living in VL endemic area in the northwest and localities in the northeast and center of the country. Of the 80 dogs, 59 presented one or several of the typical clinical signs (such as lymphadenopathy, hair loss, dermal lesions, onychogryposis, and cachexia) and 56 had showed anti-L. infantum antibodies. All of the 59 dogs with typical clinical signs were sacrificed after obtaining the consent of their owners, and Giemsa stained-smears were prepared from liver and spleen tissues.

Peripheral blood samples were collected into tubes with sodium citrate anticoagulant 4\% (Merck, Germany) for PCR testing and into tubes without anticoagulant for DAT. All samples used for serology and PCR were stored at $-20^{\circ} \mathrm{C}$ until use.

All experiments on the humans and animals were performed according to the guidelines of the Ethical Board of Tehran University of Medical Sciences, Iran.

2.1.4. Parasite Culture and Cryopreservation. All the specimens including exudates from human skin lesions and rodents ears, aspirates from bone marrow of human patients, and liver and spleen of dogs were cultured in RPMI-1640 medium (Gibco, Life technologies GmbH, Frankfurt, Germany) supplemented with $10-15 \%$ fetal bovine serum (Gibco, Germany), $100 \mathrm{U} / \mathrm{mL}$ penicillin, and $100 \mathrm{ug} / \mathrm{mL}$ streptomycin (Gibco, Germany) and incubated at $24-25^{\circ} \mathrm{C}$. Five days later the last subcultured parasites were harvested, washed in sterile phosphate buffered saline (PBS, pH: 7.2-7.4), and kept in $-20^{\circ}$ until used. All the cultures specimens were preserved in liquid nitrogen for further possible use.

2.1.5. Preparation of DAT Antigen and Performance of the Test. L. infantum (MCAN/IR/07/Moheb-gh; GenBank accession number FJ555210) was cultured in RPMI1640. The parasites were harvested at early stage of stationary phase (about $120 \mathrm{~h}$ later), washed with PBS ( $\mathrm{pH}=7.2$ ), and subjected to trypsin digestion. The parasites were stained with Coomassie brilliant blue R-250 dye (Sigma, USA), fixed with formaldehyde (Merck, Germany) $1.2 \%$, and used for DAT on humans, and dogs' sera. DAT was performed to determine the presence of the anti-Leishmania antibodies $[14,25,26]$. The titers $\geq$ $1: 3200$ and $\geq 320$ were considered positive for humans and dogs, respectively [10].

2.1.6. DNA Extraction, PCR, and RFLP. DNA was extracted from cultured parasites from human skin lesions, rodents' ears exudates, and whole blood samples of suspected VL patients and canine visceral leishmanissis dogs by using the High Pure PCR Template Preparation Kit (Roche Diagnostics 


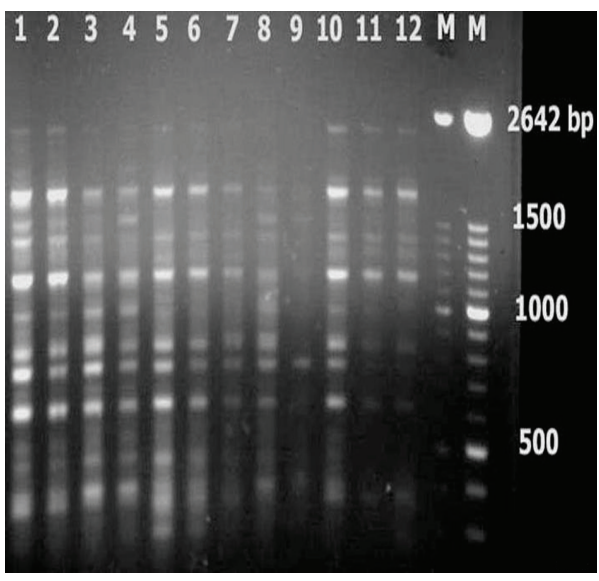

(a)

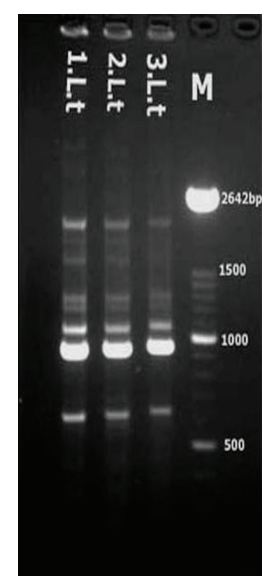

(b)

FIGURE 1: (a) Random amplified polymorphic DNA (RAPD) profiles obtained from Leishmania stocks and isolates with the AB1-O7 primer. Lane 1, L. infantum stock, lanes 2-12, L. infantum isolates. (b) Lane 1, L. tropica stock, lanes 2-3, L. tropica. M: 100 bp size marker (Roche).

GmbH, Mannheim, Germany) according to the manufacturer's instructions. Partial ITS1 of ribosomal RNA (ITSIrRNA) gene of the parasites was amplified using the primers LITSR ( $5^{\prime}$-CTGGATCATTTTCCGATG- $\left.{ }^{\prime}\right)$ and L5.8S ( $5^{\prime}$ TGATACCACTTATCGCACTT- $3^{\prime}$ ) and PCR conditions outlined by others [19]. Amounts of $10 \mu \mathrm{L}$ of amplicons were digested with Fast digestion HAEIII (BsuRI) enzyme (Fermentas $\mathrm{GmbH}$, Thermo Scientific, Germany) according to the manufacturer's instructions, and the fragments were visualized on 3\% agarose gels [22]. Leishmania species were identified based on obtained patterns alongside reference species including L. tropica (GenBank accession number EF653267), L. major (GenBank accession number JN860745), L. turanica (GenBank accession number EU395712), and $L$. infantum (GenBank accession number EU810776).

2.1.7. Variation Analyses by RAPD-PCR. RAPD-PCR was performed for 46 DNA extracted from cutaneous and visceral cultured parasites samples with the primer AB1-O7:5' GGTGACGCAG-3' and PCR conditions outlined by others $[4,27]$. This primer was chosen due to its reproducibility and its ability to reveal inter- and intraspecies variation.

Variations among isolates of different species were calculated based on profile of bands produced by AB1-O7 primer (Figure 1). A matrix for each of the isolates was constructed with the numbers " 1 " and " 0 " corresponding to the presence and absence of each possible band. For determining the polymorphism rate among different Leishmania species, we identified the bands that were common in less than $50 \%$ of the isolates (polymorphic bands) using the following equations, where $Z$ was calculated by (1) for each polymorphic band. $Z$-test was used for estimating the relative risk between two independent groups $[28,29]$. If the obtained value for $z$ in a band is less than $<1.64$ with a confidence interval of more than 95\% $(P<0.05)$, then it will be included as a polymorphic band in the matrix. After determining the polymorphic bands, polymorphism rate was calculated via (2), where $X$ is the number of isolates which contain a polymorphic band, $N$ is the total number of isolates in each group, and $M$ is the number of total bands in each isolate

$$
\begin{gathered}
Z=\frac{(X / N)-0.5}{\sqrt{(0.5 \times 0.5) / N}}, \\
\text { Polymorphism rate }=\frac{X}{M \times N} \times 100 .
\end{gathered}
$$

2.1.8. Nucleotide Sequence and Phylogenic Analyses. Partial sequences of ITS1 gene from 20 cutaneous and visceral Leishmania isolates (Table 1) were sequenced using LITSR as the forward primer. The sequences were aligned by ClustalX software, the similarities among them were calculated, and a phylogenic tree was constructed by using Tamura 3parameter option of the maximum likelihood method by MEGA 5 software.

\section{Results}

\subsection{Cutaneous Leishmaniasis}

3.1.1. Human. Among 173 suspected CL patients, 122 (70.5\%) were positive by microscopy and $112(64.7 \%)$ by culture method (Figure 2). DNA extracted from 112 culture positive samples yielded an amplicon of 300-350 bp. Digestion of amplicons with HAEIII (BsuRI) produced two profiles: 75 amplicons (67\%) produced two bands of $220 \mathrm{bp}$ and $140 \mathrm{bp}$ indicative of $L$. major, and other 37 (33\%) produced three bands of $200 \mathrm{bp}, 60 \mathrm{bp}$, and $50 \mathrm{bp}$ indicative of L. tropica (Figure 3). Almost all of the L. tropica isolates originated from northeastern areas, while L. major isolates belonged to the center, west, southern, and southwest of the country (Figure 4).

3.1.2. Rodents. Of the 60 caught rodents, 25 ( $24 R$. opimus and one $N$. indica) were parasitologically positive (microscopy and/or culture). Among the infected R. opimus, 21 harbored 


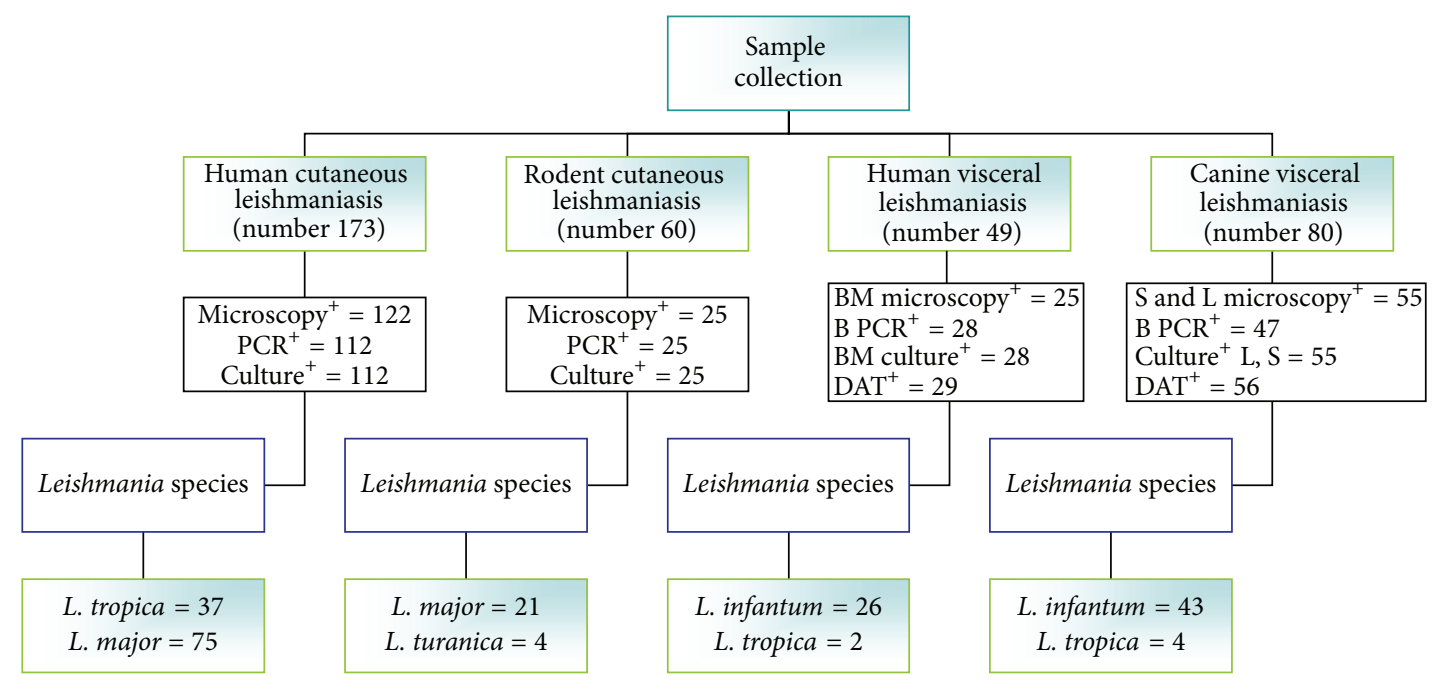

FIGURE 2: Flow chart which outlines the major parts of the study and results. B PCR: blood PCR, BM: bone marrow, S: spleen tissue, L: liver tissue, and DAT: direct agglutination test.

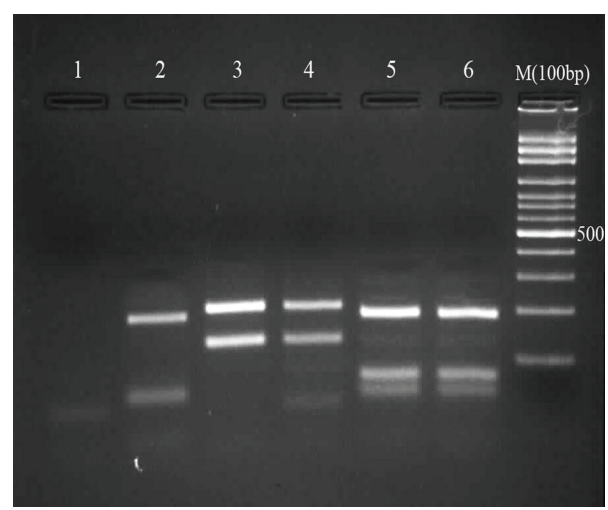

FIGURE 3: Restriction fragment length polymorphism (RFLP) patterns obtained from Leishmania stocks and patients samples. lane 1 , negative control, lane 2, L. tropica, lanes 3, 4, L. major, lanes 5, 6, L. infantum. M: 100-bp size marker (Fermentas).

L. major and 3 L. turanica. The infecting species in N. indica was L. turanica (Figure 2).

\subsection{Visceral Leishmaniasis}

3.2.1. Human. From 49 suspected VL patients, 29 (59.2\%) had anti-Leishmania antibodies and were considered positive (titers $\geq 1: 3200)$. Microscopy and culture methods identified Leishmania infection in 25 (51.02\%) and 28 (57.14\%) individuals, respectively. ITS1-PCR using the DNA from 49 patients' blood yielded the expected band only in 28 cases $(57.14 \%)$. Digestion of amplicons with HAEIII (BsuRI) yielded three bands of $200 \mathrm{bp}, 80 \mathrm{bp}$, and $60 \mathrm{bp}$ in 26 (92.86\%) isolates indicative of $L$. infantum and three bands of $200 \mathrm{bp}, 60 \mathrm{bp}$, and $50 \mathrm{bp}$ in $2(7.14 \%)$ isolates indicative of L. tropica (Figure 3).

3.2.2. Domestic Dogs. Analyses of sera from 80 domestic dogs showed that $56(70 \%)$ were positive (at titers $\geq 1: 320$ ). Whole

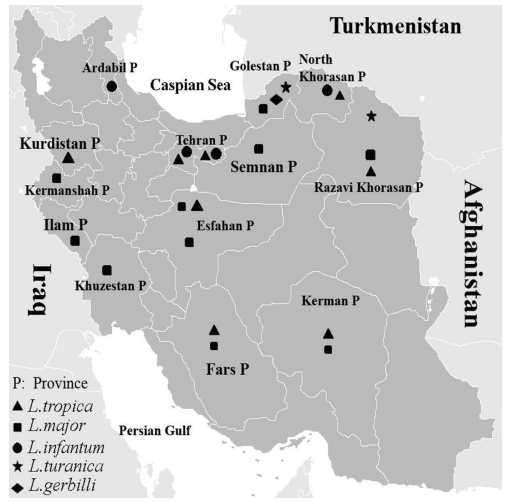

FIgURE 4: Distribution of Leishmania species isolated from human and animal hosts from some parts of Iran, identified based on ITS1PCR-RFLP (2004-2012).

blood dog DNA samples were successfully amplified in only 47 (58.75\%) cases. RFLP analyses revealed L. infantum in 43 dogs (91.5\%) and L. tropica in 4 dogs (8.5\%). Of the 59 dogs with typical symptomatic VL signs (including the 56 DAT serologically positive cases), 55 showed infection in spleen and liver with both microscopy and culture (Figure 2).

3.3. RAPD-PCR and Polymorphism Rate. The polymorphism rate among $L$. tropica isolates was $3.6 \%$. The rate among $L$. major isolates was $7.3 \%$ and among human VL isolates was $2.8 \%$. The highest rate $(9.8 \%)$ was found among canine VL isolates.

3.4. Phylogenetic Tree. The Leishmania isolates were grouped into four main clads representing $L$. major, L. turanica, $L$. infantum, and L. tropica. Within the clad, L. major was more associated with $L$. turanica than $L$. infantum. The L. tropica supports a clear divergence between $L$. tropica isolates from 


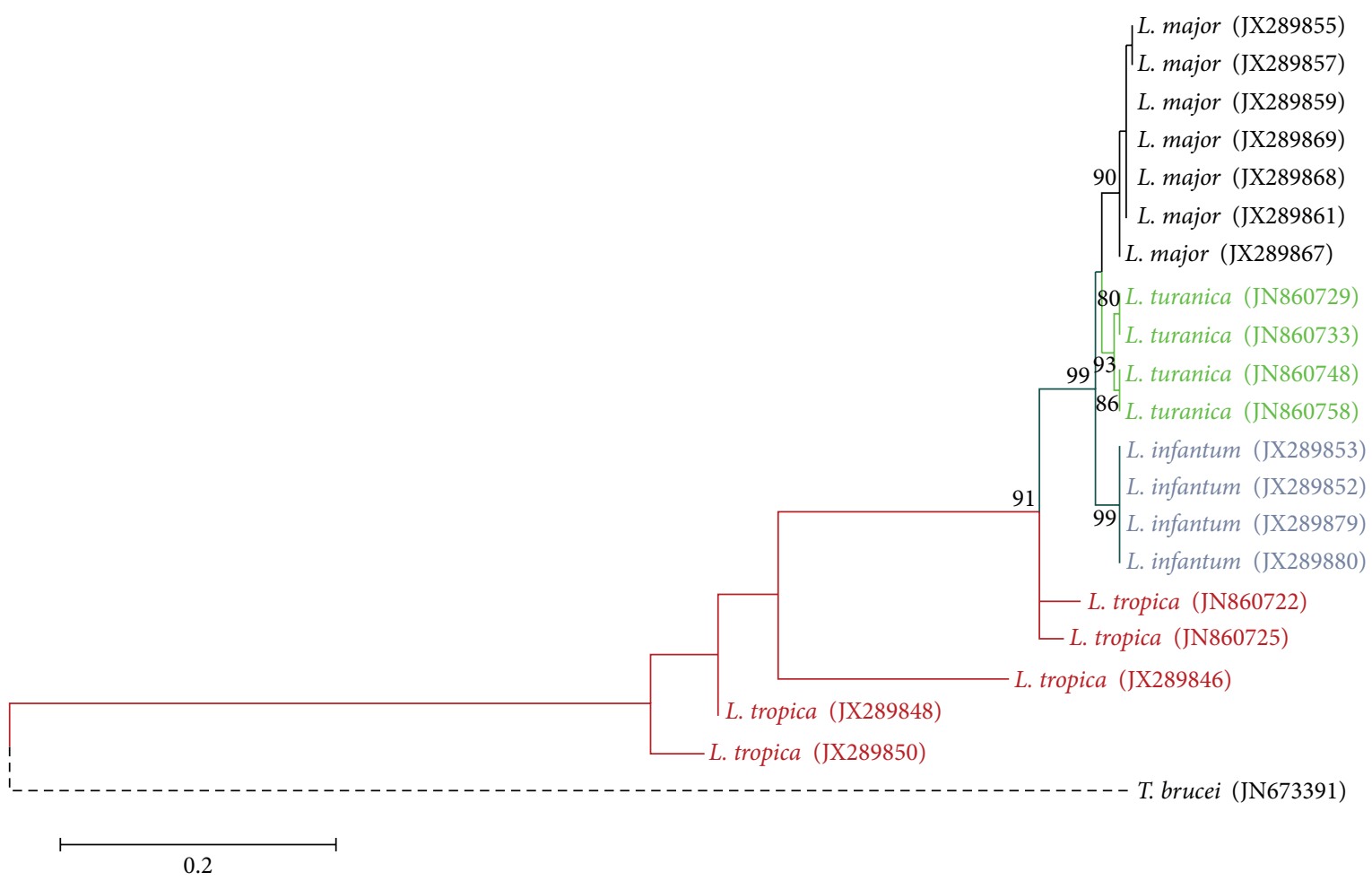

FIGURE 5: Phylogenic tree of the ITS1 region nucleotide sequences of Leishmania isolates recovered from humans and reservoir animals infected with cutaneous and visceral leishmaniasis. The tree was constructed by using the maximum likelihood, Tamura 3-parameter model in MEGA software version 5. The numbers above the branches indicate the percentage of bootstrap. Branches without numbers have frequencies of less than $70 \%$.

TABLE 1: The accession numbers of Leishmania ITS1 sequences in Iranian species submitted in GenBank.

\begin{tabular}{|c|c|c|c|c|c|c|c|}
\hline No. & Code & Place of isolation city/province & Disease & Source & RFLP/SEQ & Species & ACC.NO \\
\hline 1 & MHOM/IR/10/Esfahan-1 & Esfahan/Esfahan & $\mathrm{CL}$ & Human & $+/+$ & L. major & JX289855 \\
\hline 2 & MHOM/IR/10/Esfahan-3 & Esfahan/Esfahan & $\mathrm{CL}$ & Human & $+/+$ & L. major & JX289857 \\
\hline 3 & MHOM/IR/10/Esfahan-5 & Esfahan/Esfahan & $\mathrm{CL}$ & Human & $+/+$ & L. major & JX289859 \\
\hline 4 & MHOM/IR/10/Esfahan-7 & Esfahan/Esfahan & $\mathrm{CL}$ & Human & $+/+$ & L. major & JX289861 \\
\hline 5 & MHOM/IR/05/Kermanshah_kr3 & Kermanshah/Kermanshah & $\mathrm{CL}$ & Human & $+/+$ & L. major & JX289869 \\
\hline 6 & MHOM/IR/05/Kermanshah_kr1 & Kermanshah/Kermanshah & $\mathrm{CL}$ & Human & $+/+$ & L. major & JX289867 \\
\hline 7 & MHOM/IR/05/Kermanshah_kr2 & Kermanshah/Kermanshah & $\mathrm{CL}$ & Human & $+/+$ & L. major & JX289868 \\
\hline 8 & MHOM/IR/12/Bam2 & Bam/Kerman & $\mathrm{CL}$ & Human & $+/+$ & L. tropica & JX289846 \\
\hline 9 & MHOM/IR/12/Bam4 & Bam/Kerman & $\mathrm{CL}$ & Human & $+/+$ & L. tropica & JX289848 \\
\hline 10 & MHOM/IR/12/Bam6 & Bam/Kerman & $\mathrm{CL}$ & Human & $+/+$ & L. tropica & JX289850 \\
\hline 11 & MHOM/IR/02/M12-Khorasan & Mashhad/Khorasan & $\mathrm{CL}$ & Human & $+/+$ & L. tropica & JN860722 \\
\hline 12 & MHOM/IR/02/M39-Khorasan & Mashhad/Khorasan & $\mathrm{CL}$ & Human & $+/+$ & L. tropica & JN860725 \\
\hline 13 & MRHO/IR/11/GOL-5 & Golestan & $\mathrm{CL}$ & Rodent & $+/+$ & L. turanica & JN860748 \\
\hline 14 & MRHO/IR/10/Khorasan6 & Khorasan & $\mathrm{CL}$ & Rodent & $+/+$ & L. turanica & JN860733 \\
\hline 15 & MRHO/IR/11/GOL-19 & Golestan & $\mathrm{CL}$ & Rodent & $+/+$ & L. turanica & JN860729 \\
\hline 16 & MRHO/IR/11/GOL-15 & Golestan & $\mathrm{CL}$ & Rodent & $+/+$ & L. turanica & JN860758 \\
\hline 17 & MCAN/IR/08/Moshfe237-VL-8 & Meshkinshahr/Ardebil & $\mathrm{VL}$ & Canine & $+/+$ & L. infantum & JX289879 \\
\hline 18 & MCAN/IR/08/Moshfe240-VL-9 & Meshkinshahr/Ardebil & $\mathrm{VL}$ & Canine & $+/+$ & L. infantum & JX289880 \\
\hline 19 & MCAN/IR/11/Zare & Bomehen/Tehran & $\mathrm{VL}$ & Canine & $+/+$ & L. infantum & JX289852 \\
\hline 20 & MCAN/IR/11/Ziaee & Meshkinshahr/Ardebil & $\mathrm{VL}$ & Canine & $+/+$ & L. infantum & JX289853 \\
\hline
\end{tabular}


the other three species. The numbers above the branches indicate the percentage of bootstrap samplings. There was no clear grouping among the 20 isolates according to their geographical origin (Figure 5).

Details of the specimens sequenced and submitted to GenBank are shown in Table 1.

\section{Discussion}

In this study, we could identify four species of Leishmania including L. major, L. tropica, L. infantum, and L. turanica by ITS1 gene followed by RFLP and sequencing. The molecular results on the Leishmania isolated from human cutaneous lesions are consistent with the epidemiologic studies committed in recent decades [3]. The results showed the major causes of CL in Iran were L. major (67\%) and L. tropica (33\%). The major foci of ZCL transmission were in the northern (Golestan), northeastern near Turkmenistan's border, central (Esfahan), western (Kermanshah) near the Iraqi border, and southern (Khuzestan) provinces of Iran. The major foci of ACL transmission were in the northeast (Razavi Khorasan province) and center of Iran in the city of Bam (Kerman province) especially after the earthquake in 2003.

$L$. major was found to be the most prevalent species in $R$. opimus (the main reservoirs of ZCL) followed by L. turanica as the second agent [13]. L. turanica is incapable of infecting humans, but it causes lesions in laboratory animals [30].

In suspected VL patients, DAT showed presence of antiL. infantum antibodies in $29(59.2 \%)$ of the human cases. PCR detected Leishmania DNA in blood samples of 28 (57.14\%) individuals. RFLP analyses revealed L. infantum as the main causative agent of VL (92.86\%) and L. tropica as the secondary agent $(7.14 \%)$. This finding is in agreement with the results of other studies performed in Iran $[10,16]$. The main endemic areas of VL were Ardebil province (Meshkin-Shahr district) in the northwest and Fars provinces in the south. HIV-Leishmania coinfection has been reported recently from northeastern Iran [31].

Of the 49 bone marrow aspirates from human VL, 25 (51.02\%) showed amastigote forms of Leishmania sp. by microscopy. DAT and PCR were both positive in all the 25 human patients.

In a similar study, PCR and microscopy of bone marrow aspirates were shown to be equally sensitive in patients with microscopically confirmed VL. Moreover, PCR could detect Leishmania DNA in bone marrow aspirates in $66.7 \%$ of suspected VL patients, while microscopy of the same material was negative [32].

Serology was more sensitive than PCR in diagnosis of suspected VL dogs. As in humans, L. infantum was the main causing agent of VL in dogs $(91.5 \%)$ and L. tropica was found in $8.5 \%$ of the cases. One of the important findings of this study was molecular detection of L. tropica DNA in a considerable number of humans and dogs with VL.

In a similar study, microscopy detected parasite in liver and spleen of $93.2 \%$ of symptomatic dogs, while serology was positive in $94.9 \%$ and PCR in $76.6 \%$ of cases [17]. PCR seems to be less sensitive than microscopy or serology for the detection of Leishmania in blood of suspected VL dogs [33]. Phylogenic trees derived from the ITS1 sequences support a clear divergence between L. tropica from the other three species, but there was no clear grouping among the isolates according to their geographical origin. In RAPD-PCR assay, we used the primer AB1-07, which had the most consistency rate between different populations of Leishmania strains in different regions.

\section{Conclusions}

Characterization of Leishmania isolates collected from different parts of Iran showed that L. tropica with $3.6 \%$ polymorphisms was the primary agent in the ACL foci and $L$. major with $7.3 \%$ genetic variations was the predominant agent in the ZCL areas of Iran. Moreover, L. infantum with $2.8 \%$ genetic variations in human VL and $9.8 \%$ polymorphisms in canine VL was found in VL endemic areas of Iran. The results of this study showed that PCR-RFLP and RAPD-PCR, despite some limitations, are simple and powerful tools for the characterization and determination of Leishmania species polymorphisms.

\section{Conflict of Interests}

All the authors declare that they have no conflict of interests.

\section{Acknowledgments}

This study received financial support from Tehran University of Medical Sciences (Projects nos.: 240/3984 and 90/13462), Tehran, Iran. The authors thank Dr. B Akhoundi and Mrs. S Charehdar for their collaboration in conducting the research.

\section{References}

[1] "Control of the Leishmaniases," Report of a Meeting of the WHO Expert Committee on the Control of Leishmaniases, Geneva, switzerland, 2010.

[2] A. J. Magill, M. Grogl, R. A. Gasser Jr., W. Sun, and C. N. Oster, "Visceral infection caused by Leishmania tropica in veterans of operation desert storm," The New England Journal of Medicine, vol. 328, no. 19, pp. 1383-1387, 1993.

[3] A. Nadim, E. Javadian, M. Mohebali, and A. Zamen Momeni, "Leishmania parasites and Leishmaniases," in Epidemiology of Leishmaniasis in Iran, Tehran University Press, Tehran, 2009 (Persian).

[4] H. Hajjaran, M. Mohebali, M. R. Razavi et al., "Identification of Leishmania species isolated from human cutaneous leishmaniasis, using random amplified polymorphic DNA, (RAPD-PCR)," Iranian Journal of Public Health, vol. 33, no. 4, pp. 8-15, 2004.

[5] I. Sharifi, S. Poursmaelian, M. R. Aflatoonian et al., "Emergence of a new focus of anthroponotic cutaneous leishmaniasis due to Leishmania tropica in rural communities of Bam district after the earthquake, Iran," Tropical Medicine and International Health, vol. 16, no. 4, pp. 510-513, 2011.

[6] M. R. Shiee, H. Hajjaran, M. Mohebali et al., "A molecular and parasitological survey on cutaneous leishmaniasis patients from historical city of kashan in Isfahan province, center of Iran," 
Asian Pacific Journal of Tropical Disease, vol. 2, no. 6, pp. 421425, 2012.

[7] S. Maraghi, A. Samarbaf Zadeh, A. A. Sarlak, M. B. Ghasemian, and B. Vazirianzadeh, "Identification of cutaneous leishmaniasis agents by nested polymerase chain reaction (Nested-PCR) in shush city, khuzestan province, Iran," Iranian Journal of Parasitology, vol. 2, no. 3, pp. 13-15, 2007.

[8] N. Rahbarian, A. Mesgarian, M. Mahmoudi Rad et al., "Identification of Leishmania species isolated from human cutaneous leishmaniasis using PCR method," Journal of Research in Health Sciences, vol. 9, no. 2, pp. 48-51, 2009.

[9] S. Alimoradi, H. Hajjaran, M. Mohebali, and F. Mansouri, "Molecular identification of Leishmania species isolated from human cutaneous leishmaniasis by RAPD-PCR," Iranian Journal of Public Health, vol. 38, no. 2, pp. 44-50, 2009.

[10] M. Mohebali, "Epidemiological status of visceral leishmaniasis in Iran: experiences and review of literature," Journal of Clinical Experimental Pathology, vol. S3, article 003, 2012.

[11] S. Razmjou, H. Hejazy, M. H. Motazedian, M. Baghaei, M. Emamy, and M. Kalantary, "A new focus of zoonotic cutaneous leishmaniasis in shiraz, Iran," Transactions of the Royal Society of Tropical Medicine and Hygiene, vol. 103, no. 7, pp. 727-730, 2009.

[12] H. Hajjaran, M. Mohebali, M. R. Abaei, M. R. Oshaghi, Z. Zarei et al., "Natural infection and phylogenetic classification of Leishmania spp.infecting Rhombomys opimus, a primary reservoir host of zoonotic cutaneous leishmaniasis in northeast Iran," Transactions of the Royal Society of Tropical Medicine and Hygiene, vol. 107, no. 9, pp. 550-557, 2013.

[13] A. A. Akhavan, M. R. Yaghoobi-Ershadi, H. Mirhendi et al., "Molecular epizootiology of rodent leishmaniasis in a hyperendemic area of Iran," Iranian Journal of Public Health, vol. 39, no. 1, pp. 1-7, 2010.

[14] M. Mohebali, H. Hajjaran, Y. Hamzavi et al., "Epidemiological aspects of canine visceral leishmaniosis in the Islamic Republic of Iran," Veterinary Parasitology, vol. 129, no. 3-4, pp. 243-251, 2005.

[15] M. A. Oshaghi, Y. Rassi, L. Tajedin et al., "Mitochondrial DNA diversity in the populations of great gerbils, Rhombomys opimus, the main reservoir of cutaneous leishmaniasis," Acta Tropica, vol. 119, no. 2-3, pp. 165-171, 2011.

[16] S. Jafari, M. Hajiabdolbaghi, M. Mohebali, H. Hajjaran, and H. Hashemian, "Disseminated leishmaniasis caused by Leishmania tropica in HIV-positive patients in the Islamic Republic of Iran," Eastern Mediterranean Health Journal, vol. 16, no. 3, pp. 340343, 2010.

[17] A. Moshfe, M. Mohebali, G. Edrissian et al., "Canine visceral leishmaniasis: asymptomatic infected dogs as a source of $L$. infantum infection," Acta Tropica, vol. 112, no. 2, pp. 101-105, 2009.

[18] H. Hajjaran, M. Mohebali, A. A. Akhavan, A. Taheri, B. Barikbin, and S. Nassiri, "Unusual presentation of disseminated cutaneous leishmaniasis due to Leishmania major case reports of four Iranian patients," Asian Pacific Journal of Tropical Medicine, vol. 6, no. 4, pp. 333-336, 2013.

[19] G. Schönian, A. Nasereddin, N. Dinse et al., "PCR diagnosis and characterization of Leishmania in local and imported clinical samples," Diagnostic Microbiology and Infectious Disease, vol. 47, no. 1, pp. 349-358, 2003.

[20] M. Soto, R. Laura, M. A. Pineda et al., "Searching genes encoding Leishmania antigens for diagnosis and protection,"
Scholarly Research Exchange, vol. 2009, Article ID 173039, 25 pages, 2009.

[21] J. Alvar, I. D. Velez, C. Bern et al., "Leishmaniasis worldwide and global estimates of its incidence," PLoS One, vol. 7, no. 5, Article ID e35671, 2012.

[22] H. Hajjaran, F. Vasigheh, M. Mohebali, S. Rezaei, S. Mamishi, and S. Charedar, "Direct diagnosis of Leishmania species on serosity materials punctured from cutaneous leishmaniasis patients using PCR-RFLP," Journal of Clinical Laboratory Analysis, vol. 25, no. 1, pp. 20-24, 2011.

[23] M. A. Oshaghi, N. Maleki Ravasan, M. Hide, E. Javadian, Y. Rassi et al., "Development of species-specific PCR and PCRrestriction fragment length polymorphism assays for $L$.infantum-donovani- discrimination," Experimental Parasitology, vol. 122, no. 1, pp. 61-65, 2009.

[24] M. Mohebali, E. Javadian, M. R. Yaghoobi-Ershadi, A. A. Akhavan, H. Hajjaran, and M. R. Abaei, "Characterization of Leishmania infection in rodents from endemic areas of the Islamic Republic of Iran," Eastern Mediterranean Health Journal, vol. 10, no. 4-5, pp. 591-599, 2004.

[25] G. H. H. Edrissian, H. Hajjaran, M. Mohebali, G. Soleimanzadeh, and S. Bokaei, "Application and evaluation of direct agglutination test in serodiagnosis of visceral leishmaniasis in man and canine reservoirs in Iran," Iranian Journal of Medical Sciences, vol. 21, pp. 119-124, 1996.

[26] M. Mohebali, G. H. H. Edrissian, A. Nadim, H. Hajjaran, B. Akhoundi et al., "Application of direct agglutination test (DAT) for the diagnosis and seroepide-miological studies of visceral leishmaniasis in Iran," Iranian Journal of Parasitology, vol. 1, no. 1, pp. 15-25, 2006.

[27] S. R. Naddaf Dezfouli, M. A. Oshaghi, H. Vatandoost, E. Javadian, Z. Telmadarei, and M. Assmar, "Use of random amplified polymorphic DNA polymerase chain reaction (RAPDPCR) and ITS2 PCR assays for differentiation of populations and putative sibling species of Anopheles fluviatilis (Diptera: Culicidae) in Iran," Iranian Journal of Public Health, vol. 31, no. 3-4, pp. 133-137, 2002.

[28] S. A. Albustan, M. A. Alnaqeeb, N. Y. Murad, and A. F. AlAlawi, "Genetic variation of inbred laboratory rats by RAPDPCR," Kuwait Journal of Science and Engineering, vol. 28, no. 2, pp. 391-399, 2001.

[29] J. M. Lachin, "Relative risk estimates and tests for two independent groups," in Biostatistics Methods: The Assesment of Relative Risks, John Wily\&Sons, Toronto, Canada, 2nd edition, 2000.

[30] H. Hajjaran, M. Mohebali, S. Alimoradi, M. R. Abaei, and G. H. Edrissian, "Isolation and characterization of pathogenic Leishmania turanica from Nesokia indica (Rodentia, Muridae) by PCR-RFLP and ITS1 sequencing in Iran," Transactions of the Royal Society of Tropical Medicine and Hygiene, vol. 103, no. 11, pp. 1177-1179, 2009.

[31] R. Shafiei, M. Mohebali, B. Akhoundi, M. S. Galian, F. Kalantar et al., "Emergence of co-infection of visceral leishmaniasis in HIV-positive patients in northeast Iran: a preliminary study," Travel Medicine and Infection Diseases, vol. 13, pp. S1477-S8939, 2013.

[32] O. F. Osman, L. Oskam, E. E. Zijlstra et al., "Evaluation of PCR for diagnosis of visceral leishmaniasis," Journal of Clinical Microbiology, vol. 35, no. 10, pp. 2454-2457, 1997.

[33] C. Margonari, J. A. Menezes, M. N. Rocha et al., "Public knowledge about and detection of Canine visceral leishmaniasis in urban divinópolis, Brazil," Journal of Tropical Medicine, vol. 2012, Article ID 429586, 8 pages, 2012. 

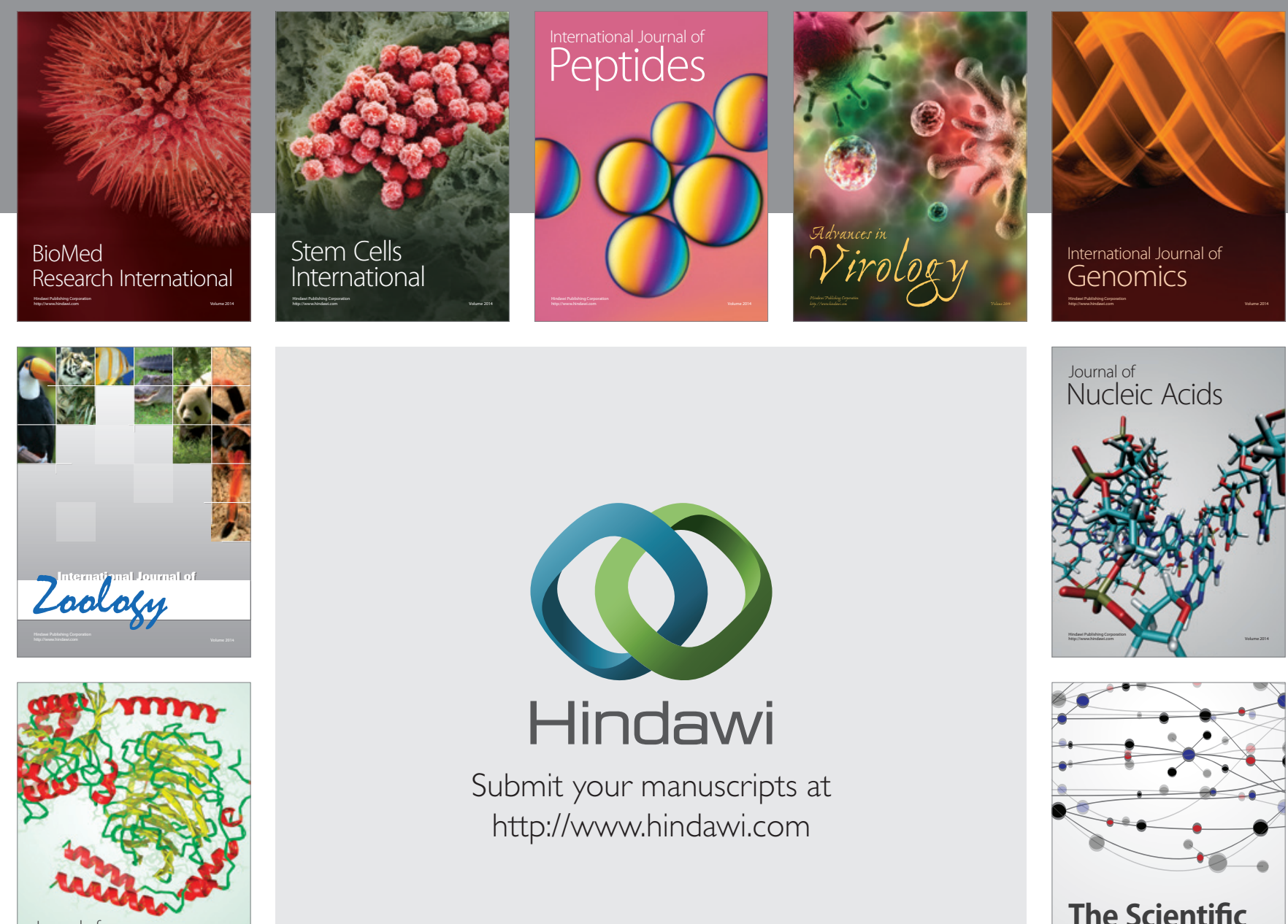

Submit your manuscripts at

http://www.hindawi.com

Journal of
Signal Transduction
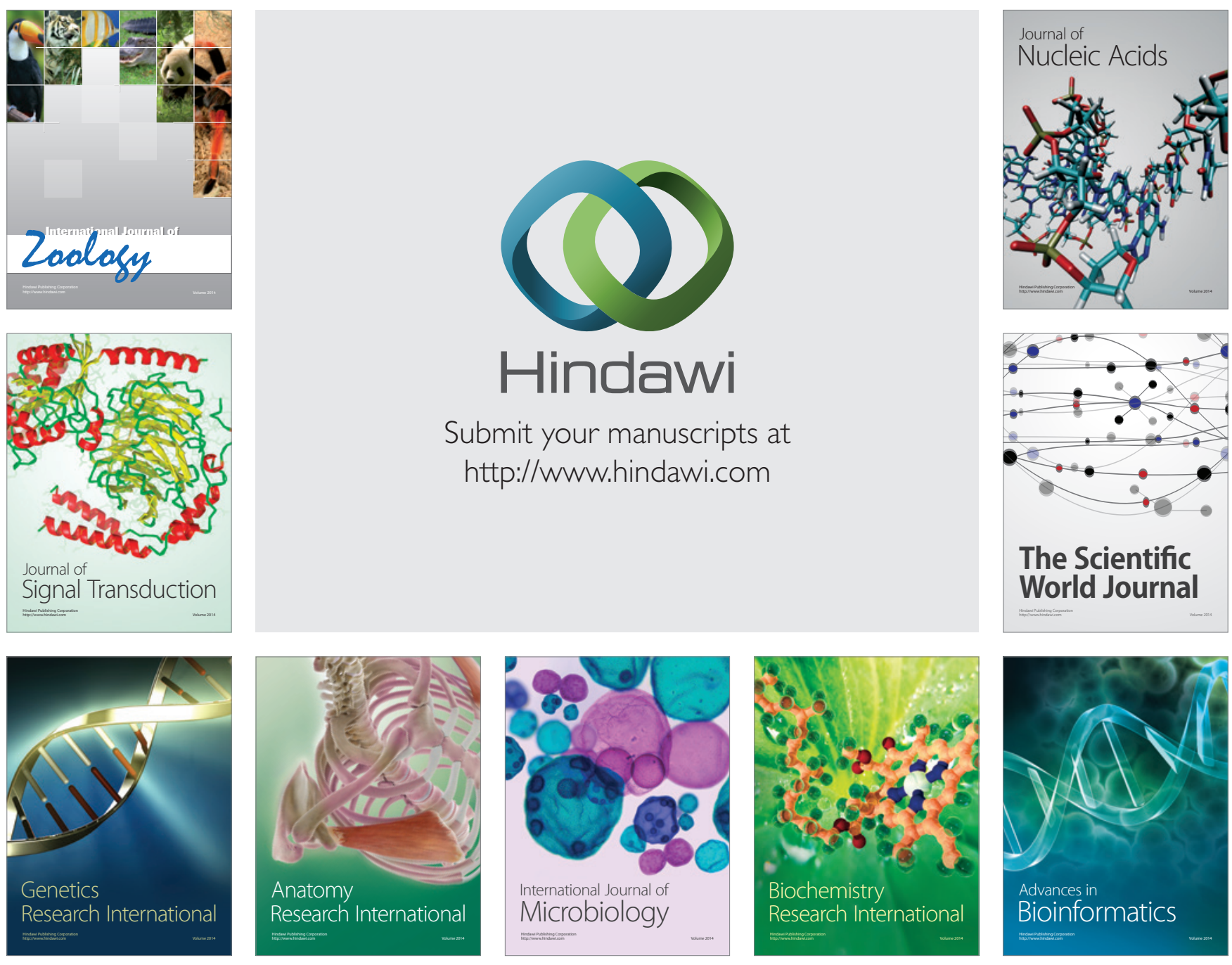

The Scientific World Journal
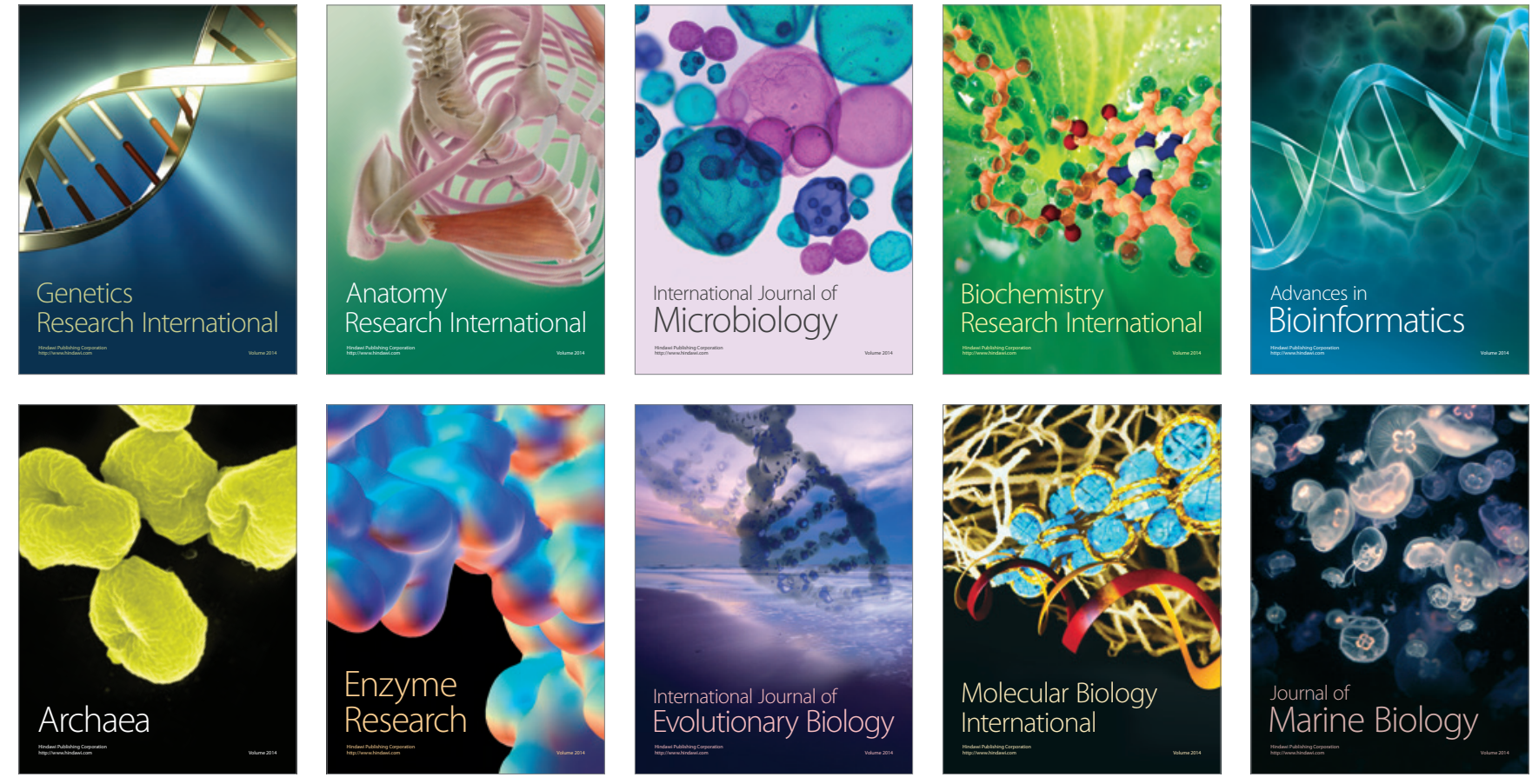